\title{
STATISTICAL MECHANICS AND THE SECOND LAW OF THERMODYNAMICS*
}

\author{
BY P. W. BRIDGMAN
}

One thing that has much impressed me in recent conversations with physicists, particularly those of the younger generation, is the frequency of the conviction that it may be possible some day to construct a machine which shall violate the second law of thermodynamics on a scale large enough to be commercially profitable. This constitutes a striking reversal of the attitude of the founders of thermodynamics, Kelvin and Clausius, who postulated the impossibility of perpetual motion of the second kind as a generalization from the uniformly unsuccessful attempts of the entire human race to realize it. Paradoxically, one very important factor in bringing about this change in attitude is the feeling of better understanding of the second law which the present generation enjoys, and which is largely due to the universal acceptance of the explanation of the second law in statistical terms, for which Gibbs was in so large a degree responsible. Statistical mechanics reduces the second law from a law of ostensibly absolute validity to a statement about high probabilities, leaving open the possibility that once in a great while there may be important violations. Doubtless another most important factor in present scepticism as to the ultimate commercial validity of the second law is the discovery of the importance in many physical phenomena of those fluctuation effects which are demanded by statistical mechanics. It is very hard indeed for one who has witnessed the Brownian motion for the first time to resist the conviction that an ingenious enough engineer might get something useful out of it, and I have no doubt that many in this audience have tried their own hand at designing such a device, and I also have no doubt that their success has been discouragingly nil.

There are other aspects also of the statistical point of view which have become prominent in the last few years, as for ex-

* The Ninth Josiah Willard Gibbs Lecture, delivered at New Orleans, December 29, 1931, under the auspices of this Society, at a joint meeting of this Society, the American Physical Society, and Section A of the A.A.A.S. 
ample, the speculations of Eddington on time's arrow and on the meaning of time in general, the speculations of Lewis as to completely reversible time, all the recent concern and new notions about the destiny of the universe as a whole, in which thermodynamic arguments play a most important part, and it is of course notorious that the notions of probability, which are fundamental in statistics, are at the very bottom of wave mechanics.

It is evident, therefore, that the statistical point of view entails consequences important both conceptually and practically. In the hope of helping a little to a better understanding of the situation I propose today to examine a few of the implications and consequences of the statistical point of view. The program is a very modest one, and I hope you will have no expectation of a final solution of any of these difficult questions; my primary purpose is to awaken a more vivid self consciousness of what the situation actually is. I shall be mostly concerned with the classical statistics, and shall have less to say about questions raised by wave mechanics; we shall find that the questions raised by the classical point of view are sufficiently fundamental. I shall throughout adopt the point of view that I have called operational, that is, I shall seek the meaning of our statements and concepts by trying to analyze what it is that we do when we are confronted with any concrete physical situation to which we attempt to apply the concept or about which we make the statement.

It is in the first place most important to realize that the statistical method, in which the notions of probability are fundamental, has, when applied to the understanding of physical situations, certain inherent, unique, logical characteristics, so that any account which statistics can give of physical phenomena must have an entirely different aspect from that of such a method of approach as classical mechanics, for example. The reason for this is that probability is not a notion which can be applied to concrete individual events, whereas we demand that we understand, or predict, or control, the individual event. I demand to know what will happen when I throw this particular stone in the air, or explode this particular charge in the cylinder of a gas engine. Ordinary mechanics gives an unequivocal answer, and in general the explanations of ordinary me- 
chanics make direct contact with just such concrete individual physical events. But the notions of probability have no such application to individual events, and in fact the notion of probability is meaningless when applied to an individual event. The proof of this is given by mere observation of what we do in applying the notion of probability. Suppose that I show you a die and remark that I intend to throw it in a minute. You volunteer the information that the probability is one-sixth that the throw will be a six. I am sceptical and ask you to justify your statement by pointing out the property of the event, when it takes place, that can be described as a probability of one-sixth for a six. I then make the throw and get a six. What possible characteristics has this single event that can justify your statement? Your statement has immediate meaning only when applied to a long sequence of events, or when applied to the construction of the die and the method of throwing it. Even when applied to a sequence of events there is always an unbridgeable logical chasm thwarting a precise application of the notion of probability to any actual sequence. Consider, for example, the classical example of tossing a coin. In practice our first concern is to determine whether the coin is a fair coin, that is, whether it is equally likely that heads or tails appear. Suppose that we make a million throws, and find the excess of one or the other not to be more than 1,000. Then we are likely to say that the coin is fair. But, logically, we are bound to recognize that the coin may have been loaded so that heads were, perhaps, nine times as likely to appear as tails, only that we had happened on one of those excessively rare sequences in which as many tails appeared as heads. Rigorously, there can be no method by which we can be sure that all our past experience has not been one of these excessively rare sequences which logically we are bound to recognize as possible in any statistical assembly.

Passing over these logical difficulties in applying precisely the notions of probability to any actual physical situation, it is evident, I think, that when applied to individual events, probability can have only a secondary or derived meaning. I believe that an examination, as the operational point of view prescribes, of what one does, will show that the meaning to be ascribed to the probability of individual happenings is to be found in the 
rules of the mental game that one plays in thinking about and trying to understand the individual events. This has important consequences when we attempt to use the notions of probability in building physical theories. We must recognize in the first place that any physical theory demands the construction of some sort of model. Now any model involving notions of probability is of necessity more remote from the physical situation and more esoteric in character than the more usual and naive models, such as are offered by ordinary mechanics. For any statistical model involves conventionalized events to which the notions of probability are by definition applicable, although the notion of probability does not apply at all to the concrete physical events which are the counterpart of the events of the model. It is therefore not surprising that the connection between the properties of the statistical model and the corresponding physical system is somewhat different from the connection in the more ordinary sorts of model.

It would be possible to digress considerably here to discuss the properties which we demand in our models, and the uses to which we put them. The subject is fairly obvious, however, and I believe we can safely assume an understanding of the essential features. The least exacting demand that we make of a model is that it serve as a calculating device, by which we can predict actual physical happenings, and for this purpose any sort of consistent correspondence between the model and the physical happening is satisfactory. The simplest way in which the statistical model can satisfy this simplest and minimum demand is evidently that actual happenings in the physical system shall correspond to high probabilities in the statistical model. This furthermore seems to be the only possibility and all that we can do; in the model there are no certainties, only probabilities, some of which, it is true, may be very. close to unity, whereas in the physical system there are invariable happenings, as for example, a cake of ice always melts when it is heated above the melting point, or the external atmosphere always rushes into an exhausted electric light bulb when it is cracked. On the other hand, it is most natural to say that low probabilities in the model correspond to infrequent occurrences in the physical system. But to go further, and specify exactly how close to unity we shall demand that the probability be that 
is to correspond to an invariable happening, is not so easy, and there seems to be a certain unavoidable fuzziness here defeating every endeavor to make sharp connection between the model and actuality. There is a still greater difficulty in giving a precise physical significance to events in the model of low probability; we shall return to this question. For the present the important point for us is that any statistical model is in peculiar degree purely a paper and pencil model and peculiar limitations may be expected in the use of such a model.

If the only demand that we put on the model were that it should serve as a computing device, the situation would be comparatively simple and could be quickly dismissed. But as a matter of fact we make the more exacting demand that the model enable us to understand the physical situation, and to this end we demand that there be a further correspondence between the properties of the model and of the physical system. Since we do not usually make the extreme demand that the model enable us to understand all the physical properties of the system at once, we usually do not demand that there be an exhaustive correspondence between the properties of the physical system and those of the model, but we are satisfied with a correspondence of those properties only which are pertinent for our immediate purposes. Thus for the discussion of the thermodynamic properties of a perfect gas, a model is usually sufficient in which the molecules of the actual gas are replaced by perfectly elastic spheres or ellipsoids, although such a model gives no hint of the optical properties of the actual gas. It is curious, however, that even for thermodynamic purposes we would not be satisfied with a model in which the number of fictitious molecules is not equal to the number of actual molecules which various other sorts of physical evidence lead us to ascribe to the actual gas.

If, now, we are attempting to find a model for the thermodynamic properties of a gas, we see that the accepted models which satisfy these additional requirements go far beyond the original demands, for in such models we encounter all the phenomena of fluctuations. Strictly, such a model never comes to equilibrium, and cannot therefore possess any property which strictly corresponds to temperature as defined classically in terms of equilibrium states. The remarkable fact, of course, 
is that the fluctuations of the model were found to correspond to fluctuations in the physical system, as shown by the Brownian motion, and in consequence we have now come to recognize that temperature is physically only an approximate concept, instead of the exact concept originally assumed in thermodynamics. This gives us at once one possible method of dealing with the second law and its apparent violations. If we choose to formulate the second law by the statement that $d Q / T$ is an exact differential, then by its very form it applies only to those situations to which the temperature concept applies, and since the temperature concept never exactly applies to any physical situation, we are left with a law which may be rigorously exact in the limit, but which applies to no actual situation. This method of meeting the situation may perhaps be satisfactory to the pure logician, but to the individual interested in filling his pockets by bootlegging entropy, such considerations will appear as uninteresting and as ineffectively legalistic as the restrictions which the more ordinary sort of bootlegger fails to recognize.

It is probably not possible to set up a mechanistic model of a purely thermodynamic system, for the thermodynamic system knows no details, but only pressure and volume and temperature, whereas it is the essence of a mechanical model that it contain details which can have no counterpart in the physical system in so far as it is purely thermodynamic. This prepares us to recognize that the concepts of thermodynamics have no absolute validity, but are relative to the operations, and in particular to the scale of the operations which we use. Thus a fluid in turbulent motion may have a temperature from the point of view of a thermometer with a bulb several centimeters in diameter, but may have no temperature from the point of view of a minute thermo-couple such as biologists have recently used in probing the interior of single cells. Or again, the entire body of phenomena to which the so-called third law is applicable would immediately appear in a different aspect and the third law would no longer be applicable if the operation of taking atoms apart and recombining them was added to the other permissible operations of a more conventional character. In recent papers Dr. David Watson has discussed some of the consequences of a recognition of some of the other relativistic characteristics of the entropy concept. 
There seem to be two diametrically opposite and equally natural reactions to an appreciation of this situation. The first reaction is that of the younger generation, part of which, at least, expects to discover some day in the realm of microscopic operations the possibility of a profitable entropy bootlegging business, and there is, secondly, the reaction of the other school, which is convinced that the second law involves something absolutely fundamental, and that any formulation in terms involving relative magnitudes or permissible operations can be only an incomplete formulation.

In the endeavor of the second school to find a more fundamental formulation it is natural to attempt to capitalize the striking success that the statistical point of view has already had in dealing with the phenomena of fluctuations. One recent and well known attempt in this direction is that of Eddington to appraise the second law in terms of what is essentially a shuffling process. Eddington sees in the universal tendency for entropy to increase, or for a system to pass from a less to a more probable configuration, the analog of what happens when a pack of cards is shuffled. Now although there may be strong points of analogy between these two processes, it seems to me that there are also essential differences, and that the analogy on the whole is not a happy one. Shuffling acquires meaning only when we are able to apprehend the cards as individuals by marking them so that we can identify them as individuals, but in such a way that there shall be absolutely no effect on the shuffling process. The picture that Eddington had in mind was a pack of cards freshly received from the manufacturer, arranged in suits and by rank in the suits, and then shuffled and losing all trace of its original arrangement. But as G. N. Lewis has remarked, the arrangement of the cards in suits is one of entirely arbitrary significance; from the point of view of some other game than whist theinitial arrangement was already a completely shuffled arrangement as well as all the subsequent ones. Or we may look at it in another way. Imagine an infinite sequence of deals, the cards being partially shuffled between each deal, and suppose that a complete record is kept of all the hands. Somewhere in this sequence there will be deals in which the cards are distributed among the hands by suit and the arrangement in each hand is by rank. If one examines the record it will be found 
that as one proceeds away from the exceptional deal, both forward and backward in the sequence, all trace of the regular arrangement becomes obliterated. That is, shuffling with respect to any configuration selected arbitrarily as of special significance is symmetrical with respect to past and future time, and the analogy with the thermodynamic situation disappears. There are other difficulties with the shuffling point of view. We have seen that shuffling has meaning only when the cards can be identified as individuals. But if the cards can be handled as individuals, they can be unshuffled, as any whist player will demonstrate to you within five seconds of picking up his hand. To see in the shuffling process something analogous to the inexorableness of the increase of entropy in nature involves the thesis that, although it may be possible to identify the elements in a physical situation, there is some restriction in nature which prevents us from treating these elements separately and sorting them out. This, it seems to me, is a hard doctrine. There is no suggestion of such a state of affairs in any picture offered by the classical mechanics, and classical mechanics was all that Eddington had in mind. Until the reason is elucidated for this surprising inability of ours to handle what we can see, I believe that the shuffling analogy must be judged only to obscure the situation rather than to clarify it.

At first glance, the possibility of understanding the strange restriction against touching what we can see is even more remote from the wave mechanics point of view than from the classical, for seeing is now to be considered as a kind of touching, namely touching with a photon. It may well be, however, that we have here the key to the ultimate solution, for it is not sufficient merely to touch, but the touch must also control; such a kind of touch doubtless requires the cooperation of a great many photons, and thus will be less possible of attainment than the touch by a single photon which is sufficient for recognition. But this point of view we do not follow further here.

Not only does Eddington see in shuffling the complete analogy of the inexorable increase of entropy, but he sees something even more fundamental, namely an explanation of the properties of time itself, and in particular why it is that time is unsymmetrical and flows only forward. This he has expressed by saying that the increase of entropy of the universe is what it is that gives direc- 
tion to time's arrow. This conception deals with such fundamental matters and has been hailed in so many quarters as being of such unique profundity, that we may be pardoned for stopping for an examination of what is involved. Eddington sees the crux of the matter in an essential difference between the equations of ordinary mechanics, including electrodynamics, and thermodynamics. The equations of ordinary mechanics and of electrodynamics, which express what Eddington calls primary law, are of such a character that the differential of time may be reversed in sign with no change in the equations, as, for example, in the equation for a falling body, $d^{2} s / d t^{2}=-g$. It is evident enough that the equation is unaltered if the sign of $d t$ is changed; the question is what is the significance of this observation? The significance that Eddington ascribes to it is that the equation is unaffected by a reversal of the direction of flow of time, which would mean that the corresponding physical occurrence is the same whether time flows forward or backward, and his thesis is that in general there is nothing in ordinary mechanical occurrences to indicate whether time is flowing forward or backward. In thermodynamic systems, on the other hand, in which entropy increases with time, time enters the differential equation as the first derivative, so that the direction of flow of time cannot be changed without changing the equation. This is taken to indicate that in a thermodynamic system time must flow forward, while it might flow backward in a mechanical system.

As thus expressed there seems to be considerable vagueness about some of the ideas; this vagueness I find in Eddington's original formulation. Such vagueness is natural and perhaps inevitable in a popular exposition, but if we are to understand from the operational point of view what is involved here, we must try to be more precise. Careful analysis has not yielded to me more than the following as an exact statement of what is involved. Imagine a closed system, and an assistant with a note book in which at a given instant of time he notes all the data necessary to characterize completely the configuration of the system. At a later instant of time he records the corresponding data in another note book. He then gives us the note books and we try from a study of them to determine which set of data was recorded at the earlier instant of time. If the system was a 
thermodynamic system we can make the decision, because the entropy increases with time, but if the system was a mechanical system we cannot decide which note book was used first, because examples could be set up for either one or the other. Eddington expresses this difference by saying that the direction of flow of time has no significance in the mechanical system.

It is, of course, true that the differential equation of the mechanical system is differently constructed from that of the thermodynamic system, but the significance of the difference does not need to be formulated as does Eddington. We must in the first place remember that the equation of the mechanical system, for example, $d^{2} s / d t^{2}=-g$, applies not only to a single system, that is to a single falling body, but applies as well to a family of systems. The equation has the property that, corresponding to every specific system, with its particle at a definite point moving with a definite velocity at a definite instant of time, another system is possible with its particle in the same position at the same instant, but with a negative velocity. This is because the equation is of the second order, and gives on integration two constants, which may be so adjusted as to give any position and any velocity at any instant of time. Imagine the second system set up; as time goes on it will trace out in reverse sequence the positions of the first system, as may be seen from the equation itself, which may be written

$$
\frac{d^{2} s}{d t^{2}}=\frac{d v}{d t}=\frac{d(-v)}{d(-t)}=-g
$$

It is this fact which makes it impossible to decide, in our example above, which note book was used first, because there is no way of telling from the entries alone whether they applied to the first or the second system. But in no case is there any question of time flowing backward, and in fact the concept of a backward flow of time seems absolutely meaningless. For how would one go to work in any concrete case to decide whether time were flowing forward or backward? If it were found that the entropy of the universe were decreasing, would one say that time was flowing backward, or would one say that it was a law of nature that entropy decreases with time? It seems to me that in any operational view of the meaning of natural concepts 
the notion of time must be used as a primitive concept, which cannot be analyzed, and which can only be accepted, so that it is meaningless to speak of a reversal of the direction of time. I see no way of formulating the underlying operations without assuming as understood the notion of earlier or later in time.

Lewis in a recent paper The symmetry of time in physics adopts a point of view in some respects similar to that of Eddington, although Lewis would certainly disclaim more than very partial agreement with Eddington. Lewis speculates about the fundamental significance of the symmetry of time in physics. His point of view takes its origin from the four-dimensional representation of events employed in relativity theory, and the consequent reduction of all propositions in kinematics to propositions in four-dimensional geometry, rather than from the form of the equations of mechanics, as in Eddington's theory. But both neglect what I believe to be the most important aspect of the situation. Both the equations giving the motion of the system and the four-dimensional representation of the motion are only a small part of the story. The equations are without significance unless the physical operations are also defined by which numerical values are assigned to the various symbols of the equations. For instance, in treating a falling body, we need in addition to the equation itself a set of directions for the use of the equation, in which it is set forth, among other things, that $s$ is the number obtained by making with a meter stick certain manipulations connected with an arbitrary origin and the instantaneous position of the falling body. Similarly in the fourdimensional representation, we must know the physical operations by which the numerical values of the coordinates are obtained which go into the four-dimensional diagram. If one examines the operations by which meaning is given to the symbols which occur in the equations or to the coordinates in the geometrical representation it will be found that the time concept has to be assumed as primitive and unanalyzable, for the operations essentially assume that the operator understands the meaning of later and earlier in time. For example, in order to find the velocity of a particle, one has to observe its position at some one instant of time and combine with this in a prescribed way the result of another observation at a later instant. If one does not intuitively understand what is meant by a later in- 
stant, there is no method of formulating the operations. The same situation is involved in specifying a thermodynamic system. One of the variables is the temperature; it is not sufficient merely to read at a given instant of time an instrument called a thermometer, but there are various precautions to be observed in the use of a thermometer, the most important of which is that one must be sure that the thermometer has come to equilibrium with its surroundings and so records the true temperature. In order to establish this, one has to observe how the readings of the thermometer change as time increases.

This point of view, that the schedule of operations by which the symbols acquire meaning is as important a part of the physical situation as the relations which are found to hold between the symbols themselves, has an important bearing on a very widely spread tendency in modern physics and science in general to see nothing as significant except the relations, and so to reduce all science to a kind of topology. It is this point of view that is at the bottom of Einstein's philosophy when he says, for example, that all that is observed is a series of spacetime coincidences, and which Eddington expresses by saying that nature may be reduced to a series of pointer readings. If one grants that the ultimate object of physics is to establish a certain sort of relation between us and the world of our senses, to speak with a certain monstrous naivété, I do not see how it is possible to discard as irrevelant the fact, for example, that the fourth coordinate in the four-dimensional geometry of relativity has to be obtained by an entirely different sort of operation from the other three coordinates, or to regard the entire situation as exhaustively characterized by the relations between the numbers, irrespective of how they are obtained.

We return now to a further consideration of our statistical model and the methods by which we shall establish a correspondence between its properties and those of the physical system. Hitherto we have been gratifyingly successful; events in the model of overwhelmingly high probability correspond to invariable happenings in the physical system, and less common events in the model, such as fluctuation phenomena, are found to be prophetic of a previously unsuspected new domain of physical effects, typified by the Brownian motion. Encouraged by this success, it is natural to think that we have got hold of 
something real, whatever that may mean, and to push our scheme of correlation to the logical limit, and say that all the excessively rare events corresponding to low probabilities in the statistical model are correlated with corresponding rare events in the physical system. Now it is a consequence of the fundamental assumptions which have gone into the usual statistical model, namely that all elementary configurations are entirely independent of each other, so that the probability of any configuration is to be calculated by purely combinatorial methods from the relative number of ways in which the configuration can be realized, that there is some chance of the occurrence of any configuration, no matter how unusual its properties. This would mean that in the corresponding physical system any configuration whatever, compatible with the fixed conditions, would occur occasionally, as, for example, the gas in a box will occasionally automatically all collect itself into one end. This conclusion is indeed taken literally by many experts in statistical mechanics, and in the literature statements are not uncommon, such, for example, as that of Bertrand Russell in a recent magazine article that if we put a pail of water on the fire and watch it for an indefinite time, we shall eventually be rewarded by seeing it freeze. It seems to me that there are a couple of objections that can be made to the conventional treatment of rare occurrences, which I shall now examine.

The first difficulty is with the technical method of calculating the chances of observing a rare configuration, and is concerned only with the model itself, and not with the physical application of the results of the calculations. In computing the chance of any configuration, it is always assumed that the elements of the statistical model are without influence on each other, so that the chance of a given configuration is given by merely enumerating the number of complexions corresponding to the given configuration. For example, in the kinetic theory of gases it is assumed that the location of any molecule and its velocity is, except for the restriction on the total energy and the total volume, independent of the location or the velocity of any or all of the other molecules. It may be proper enough to postulate this for the model, but we know that it cannot rigorously correspond to the physical system, for the molecules of a gas do interact with each other, as shown by the mere fact that they conserve 
their total energy, and the transmission of energy from one molecule to another takes place only at a finite rate, so that if, for example, at one instant all the velocity were in a single molecule, we would find that immediately afterward only molecules in the immediate vicinity had any velocity. This means that the assumption of complete independence must be recognized to be only an approximation, and some way of handling this approximation must be devised. The method usually adopted is to cast the problem in the form of inquiring how many observations must be made in order that the chance of observing the desired rare configuration may be one half, for example, choosing the time between observations so long that at each observation all appreciable trace of the previous configuration shall have been obliterated, so that the assumption of independence may apply. The point now is this: the time that one has to wait for the probable obliteration of all traces of a previous configuration becomes longer the rarer the previous configuration; obviously it takes longer for a gas to efface all trace of having been all concentrated in one half of its available volume than to efface the traces of a small local concentration. The situation is, therefore, that not only must we make an increasingly large number of observations in order to hope to witness a rare configuration, but the interval between our observations must also get longer. It is merely the first factor which is usually considered; when both factors are considered it is not at all obvious that the process is even convergent. This point should be subjected to further examination.

There is another difficulty connected with the mere calculation of the probability of rare occurrences presented by quantum theory. All classical calculations assume that the molecules have identity. But the uncertainty principle sets a limit to the physical meaning of identity. It is not possible to observe the position and velocity of any molecule with unlimited precision, but there is a mutual restriction. After an observation has been made, the domain of uncertainty in which the molecule is located expands as time goes on. If the domains of uncertainty of two molecules overlap, then the identity of the molecules is lost, and a subsequent observation will not be competent to decide which molecule is which. The only way of maintaining the identity of the molecules is by making observations at 
intervals so frequent that the domains of uncertainty have not had time to overlap. But this time is obviously much shorter than the time between observations demanded by the requirement that all trace of the previous configuration shall have been wiped out. Furthermore, the act of observation, by which the concept of identity acquires meaning, alters in an uncontrollable and unpredictable manner the motion of the molecules, whereas the statistical treatment requires that the molecules be undisturbed between successive observations. It seems, therefore, that the physical properties of actual molecules as suggested by quantum theory are different from those of the molecules of the model, and this would seem to demand at least designing new methods of calculating the chances of rare occurrences.

Apart from these objections, which may be met by the discovery of new theoretical methods of attack, it seems to me that the most serious difficulty with this question of rare states is met in the process of transferring to any actual physical system conclusions based on a study of the corresponding model. Suppose, for example, that we are discussing the problem of the tossing of some particular coin. If the coin is a fair coin, that is, if the chances of heads and tails are even, then our statistical model consists merely of a sequence of one or the other of two events, each of which is as likely to occur at any time as the other, absolutely independently of what may have happened elsewhere in the sequence. The theoretical discussion of this model is very easy, and we are convinced that conclusions drawn from a discussion of the model will apply to the tossing of the coin, always provided that the coin is a fair coin. As a particular problem we may consider the chance of throwing heads ten consecutive times. The chance of this is $(1 / 2)^{10}$, or $1 / 1024$, which means that in every 1,000 consecutive throws the chances will be roughly even that there will be somewhere a sequence of 10 heads. * But 1,000 throws are a good many, and it may be that we have never made so many throws, and are content merely to make the prediction that if some one else should make so many throws it would be found to be as we say. But suppose that some one questions the fairness of the coin,

* I am much indebted to Mr. H. M. James for a rigorous solution of the interesting problem involved here. He finds that between 1422 and 1423 throws are necessary for a 0.5 chance of ten or more consecutive heads. 
and says that he has reason to think that there is a bias of $10 \%$ in favor of throwing tails, so that the chance of a head at a single throw is only 0.45 instead of 0.50 . We find now on making the calculation that we shall have to make roughly 10,000 throws in order to have an even chance of getting a sequence of 10 heads; and, in general, that slight imperfections in the fairness of the coin make very large differences in the chance of rare occurrences. In view of this, we feel that it behooves us to make some objective test of the fairness of the coin before we venture to publish our prediction that we are likely to get a sequence of 10 heads in 1,000 throws. We make the most direct test possible by appealing to the fundamental definition of fairness, which is that in a large number of throws the ratio of the number of heads to tails tends to equality. But how many throws are necessary to establish such an equality with satisfactory assurance! There is another theorem here, namely that in $n$ throws the chances are even that we shall have an excess either of heads over tails or of tails over heads of $0.6745 n^{1 / 2}$. Neglecting the numerical factor for our rough purposes, this means that if we make a hundred throws the chances are nearly even that the number of heads is somewhere between 46 and 54. To establish the fairness of the coin we would have to make a considerable number of 100 throws at a time and observe whether or not the number of heads clusters between 45 and 54 . If, on the other hand, there is a $10 \%$ bias in favor of tails, the number of heads will cluster between 40 and 50 . The precise number of sequences of 100 throws at a time necessary to convince us that there is no $10 \%$ bias in favor of tails obeys no definite criterion, but it is certainly of the order of ten or more, which makes 1,000 or more throws altogether. But this was the number of throws necessary to obtain one of the rare sequences of 10 heads.

The conclusion from all this is plain; in order to establish with sufficient probability that the actual physical system has those properties which are assumed in estimating the frequency of rare occurrences it is necessary to make a number of observations so great that the probability is good that the rare occurrence has already been observed. In other words, purely logical statistical considerations never can justify us in predicting events so rare that they have never yet been observed. A pail of water has never been observed to freeze on the fire; statistical 
considerations give us no warrant whatever for expecting that it ever will. Such predictions can be made only on the basis of considerations other than statistical. Thus in the case of the coin, an exact measurement of its dimensions and of the degree of homogeneity of its metal might convince us that the chances of heads and tails were even, because of our knowledge of the laws of mechanics. But when we come to the molecules of a gas or the elements of other physical systems to which the statistical method of treatment is usually applied, we see that there is no method of independently handling the elements, so that the statistical method of testing the validity of our assumptions is the only possible one. This is a most natural situation, because if we were capable of dealing with the elements of the physical system as individuals we could apply more powerful methods than the statistical. Incidentally we may remark how very insensitive the statistical method is in studying elemental properties; this is shown by the example of the coin above, where we had to make something of the order of 1,000 throws to establish an asymmetry of $10 \%$. In many cases, however, the statistical method is doubtless the ultimate and the only method.

Another of the applications of statistical ideas in which there has always been much interest, and especially lately, is to the problem of the ultimate fate of the universe. It is a very common opinion that the second law, in its original classical form, demands the ultimate heat death of the universe, because of the inexorable increase of entropy to a final maximum, when all temperature differences shall have been wiped out. The chief mechanism in the ultimate equalization of temperature is obviously the radiation that is continually emitted by the stars. The human mind has, however, shown itself curiously unwilling to accept the prospect of a heat death, and there have been a number of attempts to avoid such an unwelcome conclusion. At least two of these, somewhat similar to each other, are based on the statistical interpretation of the second law. The first of these utilizes the theorem that in a closed mechanical system any configuration, once experienced, is bound to recur after the lapse of sufficient time. According to this view, the universe endlessly goes through cycles of repetition, the so-called Poincaré-Zermelo cycle, of prodigious but calculable duration. The obvious objection to this picture is that in order to realize a 
Poincaré-Zermelo cycle the laws of classical mechanics would have to be satisfied with an exactness quite fantastic, hopelessly beyond the possibility of direct or indirect verification. The second attempt to make statistics avoid the heat death rests on the theorem that a statistical assembly is never in complete equilibrium, but is always subject to fluctuations, and these fluctuations may attain any intensity if we only wait long enough. The present state of the universe is then to be regarded only as a fluctuation, with the possibility that similar fluctuations may recur in the future. The difficulty with this point of view is the excessive rareness of the sort of fluctuation corresponding to the present state of the universe compared with the approximate dead level of the heat death. The previous considerations apply; this is one of those configurations so rare that one has no right to predict its occurrence unless it has been previously observed. One might predict from purely statistical considerations the occurrence of such a fluctuation in the future if one were sure that one were observing such a fluctuation now. But where is the evidence for this? According to the astronomers the fluctuation has been taking the last $10^{16}$, or perhaps now $10^{10}$, years or so to smooth itself out to its still considerable roughness, and there is certainly no evidence that before $10^{16}$ years ago the entropy was decreasing instead of increasing.

There are other objections to an application of the second law to the entire universe. The original formulation of the second law was, of course, restricted to isolated systems. By what logical right can the argument be extended to the entire universe? A natural reply is that relativity theory seems to demand that the universe is finite, so that the whole universe becomes the sort of isolated system demanded by the classical formulation. But I believe that examination will nevertheless show a very important difference betweeen the smaller and the all-embracing closed system. Statistical mechanics, if it is to avoid the difficulties already discussed when applied to any individual physical situation, must make the assumption of molecular chaos. But what in the physical situation gives rise to molecular chaos? If we imagine a gas, for example, in a perfectly reflecting enclosure, and suppose that the molecules are perfectly elastic spheres, then, according to the classical picture, every collision takes place under perfectly definite conditions, so that a mathe- 
matician of sufficient power could compute backward from the present configuration to the configuration at any past time, as, for example, when a partition might have been removed from the middle of the compartment. This sort of condition certainly cannot be described as molecular chaos. But the walls are molecular in structure, so that the reflection of the individual gas molecules follows no definite rule. If we regard the molecules in the wall as part of the external universe, and if there is no coordination between the motion of the molecules of the wall and what is taking place in the gas because of the enormous magnitude of the external universe compared with the gas inside, then a physical reason justifying the assumption of molecular chaos is at once apparent. When the entire universe is considered there can be no such justification as this for assuming molecular chaos, but the whole course of events must, from the classical point of view, run a rigorously deterministic course, to which statistical considerations do not apply. It may be objected to this argument that a gas in contact with its walls in the way described above is not an isolated system. It is, of course, not completely isolated, but it is nevertheless as far as the thermodynamic requirements go, which are concerned only with transfers of energy and of heat from the outside. Complete isolation would seem to be incompatible with molecular chaos.

It was intimated at the beginning of this discussion that the heat death is supposed to be a consequence of the second law, and that the continuous enormous radiation into empty space of the stars is the most striking manifestation of this tendency. This point of view sees in the emission of every photon by a star part of the inexorable increase of entropy. I believe, however, that this is fallacious, that the relations are different, and that the heat death with which we are confronted as a consequence of continued radiation is an affair of the first law, not of the second, and will take place when all the energy of the universe has been radiated away, not merely when the energy is uniformly distributed. Elementary considerations justify this contention. Consider a body radiating into empty space. It is continually dropping in temperature and losing in energy. The emission of radiation therefore decreases the entropy of the radiating body. Consider next a body in thermal equilibrium with its surroundings; since it is in equilibrium its entropy is constant, 
and furthermore it absorbs as much radiation as it emits. Absorption of radiation, therefore, increases the entropy of the absorbing body. This is sufficient to give a straightforward account of entropy changes in radiation problems. Consider two bodies confronting each other in a cavity at constant temperature. A photon leaves one of the bodies, decreasing the entropy of that body. During the passage of the photon across the space separating the two bodies the entropy is to be thought of as associated with the photon and residing somewhere in the intermediate space. When the second body absorbs the radiation, the photon with its entropy disappears from empty space and increases the entropy of the absorbing body. At all stages of the process the entropy of the entire system is unchanged. But now suppose that one body radiates to another at lower temperature. This process is irreversible and is accompanied by an increase of entropy. The first two steps of the process, emission of the photon and passage across the intermediate space, are the same as before, and are therefore accompanied by no net change of entropy. It is only the last stage of the process, absorption by the body at lower temperature, that can give the uncompensated increase of entropy. The mechanism of this increase is to be found in a diffusion of the energy of the photon into the greater number of degrees of freedom corresponding to the lower temperature. Actually, this argument is over-simplified, and must be modified by a consideration of the distribution of the photons through the spectrum, but the details of this point of view need not concern us further. The immediate point for us is that emission of radiation into empty space is not an entropy changing process; the increase of entropy can occur, if it occurs at all, only during the act of absorption.

But what physical evidence have we of the absorption of the light radiated by the stars? To save the situation we must postulate absorption under completely unknown conditions. But is the assumption of such unknown conditions in regions so terribly far beyond access to us in order to save the second law any easier as an intellectual feat than the assumption of other unknown conditions which would defeat the second law? Are we not completely in the dark here, and had we not better admit it?

Finally, I briefly summarize what I believe to be the principal results of this analysis and indicate the possible lines of future 
progress. The most important result will be, I hope, a keen realization that in using statistics we are only using a paper and pencil model, which has logical difficulties within itself and difficulties of application to concrete physical situations which are very much greater than the corresponding difficulties with more ordinary sorts of model. Some of these difficulties I believe can never be surmounted, so that the statistical model can never be satisfactorily used by extrapolation either into remote epochs of time, to predict rare events, or into remote reaches of space, to give us an idea of the course of universal evolution. Our model has not given us a satisfactory answer to our initial question as to the possibility of commercially profitable violations of the second law. The answer to this question will probably be found when the wave mechanics point of view has been completely worked out. Some of the other logical difficulties of the classical statistics, I believe, will also be surmounted by the adoption of the wave mechanics point of view, which assumes probability to be a primitive property of the elements of the model, rather than an emergent property resulting from the cooperation of great numbers. Before, however, the wave mechanics thesis of the primitive character of the notion of probability can be accepted, much more experimental work is necessary. If the thesis is supported, as seems probable from the evidence now at hand, this will constitute to a certain extent a defeat of the purpose of the classical statistics, which was to explain why many physical assemblages of large numbers of elements obey the rules of probability. But even granted that the primitive character of the notions of probability acquires an experimental verification, it seems to me that some of the logical difficulties will persist, justifying a doubt as to the possibility of ever setting up a logically completely satisfying correspondence between our models and our experience.

HaRVARd University, Research Laboratory of Physics 